\title{
Prevalencia de anemia en niños de 1 a 4 años de edad en Asunción y Central, Paraguay 2017
}

\author{
Prevalence of anemia in children aged 1 to 4 years in \\ Asunción and the Central Department, Paraguay 2017
}

\author{
Fernando Galeano ${ }^{\oplus}$, Gabriela Sanabria ${ }^{\infty}$, Marta Sanabria ${ }^{1}$, Aníbal Kawabata ${ }^{1,2}(\mathbb{D}$, Gloria

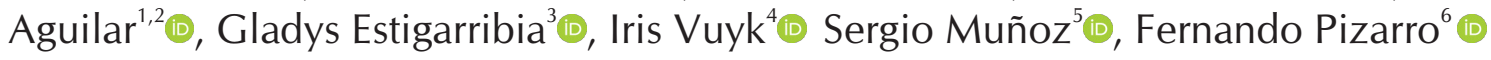 \\ ${ }^{1}$ Universidad Nacional de Caaguazú. Facultad de Ciencias Médicas. Coronel Oviedo, Paraguay. \\ ${ }^{2}$ Universidad Nacional de Caaguazú. Facultad de Ciencias Médicas. Coronel Oviedo, Paraguay. \\ ${ }^{3}$ Instituto Regional de Investigación en Salud. Coronel Oviedo, Paraguay. \\ ${ }^{4}$ Universidad Nacional del Este. Facultad de Medicina. Centro de Investigaciones Médicas. Minga Guazú, \\ Paraguay. \\ ${ }^{5}$ Universidad de la Frontera. Temuco, Chile. \\ ${ }^{6}$ Universidad de Chile. Instituto de Nutrición y Tecnología de los Alimentos. Chile.
}

\section{RESUMEN}

Introducción: Los niños constituyen el grupo más afectado por la anemia. En Paraguay no existen datos actualizados sobre prevalencia de anemia en menores de 5 años. Objetivos: Determinar la prevalencia de anemia en niños previamente sanos de 1 a 4 años que acuden a consultorios de servicios de salud y guarderías de Asunción y Central de Paraguay, durante el año 2017. Materiales y Métodos: Estudio prospectivo, de corte transverso, observacional, descriptivo. Muestreo aleatorio, estratificado. Nivel de confianza: $95 \%$ Error de muestra: 5\%. Variables: edad, sexo, hemoglobina, índices hematimétricos, ferritina, Proteína C reactiva (PCR), albúmina, zPeso/Edad, zPeso/Talla, zTalla/Edad, peso al nacer. Fueron analizadas medidas paramétricas y no paramétricas, $\chi^{2}$. La anemia se definió por criterios OMS para el grupo de 1 a 5 años de edad: Hemoglobina $\mathrm{Hb}<11 \mathrm{~g} / \mathrm{dL}$ y Volumen Corpuscular Medio VCM $<72$ fL Se utilizó el Programa Anthro v 3.2.2 y SPSS 16.0. Aprobado por Comité de Ética del Instituto de Medicina Tropical, se obtuvo consentimiento informado. Nivel de significancia $<0,05$. Resultados: Fueron reclutados 806 pacientes. Se determinó $\mathrm{Hb}, \mathrm{VCM}$ y ferritina en 706 pacientes.327 (46,3\%) fueron varones. El promedio de $\mathrm{Hb}$ fue $11,9 \pm 1,03 \mathrm{~g} / \mathrm{dL}$. Volumen corpuscular medio promedio

\section{ABSTRACT}

Introduction: Children are the group most affected by anemia. In Paraguay there are no updated data on the prevalence of anemia in children under 5 years of age. Objectives: To determine the prevalence of anemia in previously healthy children aged 1 to 4 years who presented to health services clinics and nurseries in Asunción and the Central Department, Paraguay, during the year 2017. Materials and Methods: This was a prospective, cross-sectional, observational descriptive study. We used random, stratified sampling. Confidence level: 95\% Sample error: 5\%. Variables: age, sex, hemoglobin, hematometric indices, ferritin, C-reactive protein (CRP), albumin, z Weight / Age, z Weight / Height, z Height / Age, birth weight. Parametric and non-parametric measures, $\chi 2$, were analyzed. Anemia was defined by WHO criteria for the 1 to 5 year-old group: Hemoglobin $\mathrm{Hb}$ $<11 \mathrm{~g} / \mathrm{dL}$ and Mean Corpuscular Volume MCV $<72$ fL The Anthro Program v 3.2.2 and SPSS 16.0 were used. The study was approved by the Ethics Committee of the Institute of Tropical Medicine, and informed consent was obtained. Significance level $<0.05$. Results: 806 patients were recruited. $\mathrm{Hgb}, \mathrm{MCV}$ and ferritin were determined in 706 patients. 327 (46.3\%) were male. The mean Hgb was $11.9 \pm$

Correspondencia: Marta Cristina Sanabria Correo: marta.sanabria@gmail.com

Conflicto de interés: Los autores declaran no poseer conflicto de interés.

Financiamiento: Este proyecto fue financiado por el CONACYT a través del Programa PROCIENCIA con recursos del Fondo para la Excelencia de la Educación en Investigación FEEI.

Recibido: 19/04/2021 Aceptado: 18/06/2021

DOI: https://doi.org/10.31698/ped.48022021006

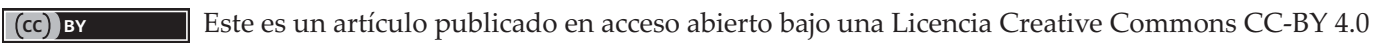


$71,2 \pm 26$ fL. Se encontraron $83 / 706$ pacientes con anemia $(11,8 \%), 44 / 83$ (53\%) fueron microcíticas (VMC $<72$ fL). PCR negativa $640 / 706(90,7 \%)$ pacientes. El promedio de albuminemia fue 4,5 $\pm 0,2 \mathrm{~g} / \mathrm{dL}$. El promedio de $\mathrm{z}$ Peso/Edad fue $0,29 \pm 1,33, z$ Peso/Talla $-0,38 \pm 1,52$ y el z Talla/Edad fue 0,92 $\pm 1,64$. Ferritina $<12 \mu \mathrm{g} / \mathrm{L}$ fue del 17,6 \%. No se encontró relación entre el peso al nacer y la anemia [p=0,11. RR: 1,45 $(0,6-2,3)]$. La prevalencia de anemia en el grupo de 12 a 23 meses fue del $39 \%$ vs el 6,9\% en preescolares $\left(\chi^{2} p<0,05\right)$. Conclusiones: La prevalencia de anemia en lactantes de Asunción y Central constituye un problema de salud pública.

Palabras claves: Anemia, ferritina, niños.

\section{INTRODUCCIÓN}

La anemia infantil es un problema de salud pública e indicador de mala nutrición, principalmente en los países en desarrollo $^{(1)}$. La Organización Mundial de la Salud considera que una prevalencia de anemia en niños entre 5 a 19,9\% constituye un problema leve, entre 20 a 39,9\% moderado y mayor al $40 \%$ grave $^{(2)}$. La prevalencia de anemia en Paraguay en el año 2004 , en menores de 5 años de edad fue del $22 \%{ }^{(3)}$.

La deficiencia de hierro y la anemia dejan una cicatriz en el desarrollo mental infantil, causando una disminución de 7 a 12 puntos en la escala de desarrollo en por lo menos tres estudios longitudinales en niños ${ }^{(4)}$. La anemia y la deficiencia de hierro (aún sin anemia) comprometen la atención y la concentración, teniendo consecuencias adversas en el periodo escolar ${ }^{(5)}$. Varios investigadores demostraron un incremento de la respuesta cognitiva en escolares y preescolares antes y después de mejorar la deficiencia de hierro ${ }^{(6)}$.

Los costos sociales asociados con la anemia en la primera infancia son altos y afectan en gran medida a los niños nacidos en grupos de bajos ingresos. Plessow et al. estimaron que más del uno por ciento del producto interno bruto (PIB) de la India se pierde cada año debido a la anemia por deficiencia de hierro entre los niños de 6 a 59 meses de edad ${ }^{(7)}$.

Es muy importante conocer la prevalencia de anemia para desarrollar estrategias de prevención de anemia en lactantes y escolares ${ }^{(8)}$.
$1.03 \mathrm{~g} / \mathrm{dL}$. Mean corpuscular volume $71.2 \pm 26$ fL. 83/706 patients with anemia $(11.8 \%)$ were found, $44 / 83(53 \%)$ were microcytic (MCV <72 fL). Negative CRP 640/706 (90.7\%) patients. Average albuminemia was $4.5 \pm 0.2 \mathrm{~g} / \mathrm{dL}$. The mean of $z$ Weight / Age was $0.29 \pm 1.33, z$ Weight / Height $0.38 \pm 1.52$ and the $z$ Height / Age was $0.92 \pm 1.64$. Ferritin $<12 \mu \mathrm{g} / \mathrm{L}$ was $17.6 \%$. No relationship was found between birth weight and anemia [ $p=0.11$. RR: $1.45(0.6-2.3)]$. The prevalence of anemia in the 12 to 23 month group was $39 \%$ vs $6.9 \%$ in preschool children $(\chi 2 \mathrm{p}<0.05)$. Conclusions: The prevalence of anemia in infants from Asunción and the Central Department constitutes a public health problem.

Key words: Anemia, ferritin, children.

\section{OBJETIVO}

Determinar la prevalencia de anemia en niños previamente sanos de 1 a 4 años que acuden a consultorios de servicios de salud y guarderías de Asunción y Central de Paraguay, durante el año 2017.

\section{MATERIALES Y MÉTODOS}

Estudio observacional prospectivo, descriptivo de corte transverso. Muestreo aleatorio, estratificado. Cálculo de tamaño muestral para un Nivel de confianza del 95\% y Error de muestra del 5\%. Efecto de diseño de 1,4. Nivel de significancia $<0.05$.

Se incluyeron 806 niños y niñas de 12 a 59 meses de edad que acudieron a guarderías y al ambulatorio de pediatría para control de crecimiento y vacunación de los hospitales públicos de Asunción y del Departamento Central durante el año 2017. Se excluyó a los niños y niñas con desnutrición o con enfermedades crónicas o congénitas.

Previo consentimiento informado de los padres o encargados se recolectaron los datos demográficos y el peso de nacimiento y se midió el peso y la talla según normativas del Ministerio de Salud Pública y Bienestar Social.

Se determinaron los datos laboratoriales de hemoglobina, hematocrito, Volumen Corpuscular Medio VCM, Hemoglobina Corpuscular Media, Concentración de Hemoglobina Corpuscular 
Media, ferritina, proteína $C$ reactiva (PCR) y albuminemia.

La determinación de estos parámetros se realizó en un laboratorio de referencia con un Contador Hematológico (Huma Count 30TS, Human Diagnostics World wide). Se utilizó sangre total colectada en un tubo Vacutainer con EDTA como anticoagulante para $1 \mathrm{ml}$ de sangre. Inmediatamente después de obtenida la muestra de sangre se agitó suavemente por inversión o en agitador mecánico suave, por al menos 5 minutos, hasta asegurar la mezcla sangre-anticoagulante. Luego de agitada la muestra se colocó directamente en la aguja de aspiración del equipo que toma $25 \mathrm{uL}$ de sangre y en forma automática se realizaron las distintas diluciones hasta entregar los resultados impresos en un formulario estándar. Se realizó control de la calidad de los parámetros hematológicos a través de un control externo (RIQAS).

Se clasificó la anemia según los criterios de OMS en: normal $(\mathrm{Hb} \geq 11,0 \mathrm{~g} / \mathrm{dL})$; anemia leve (Hb entre 10,0 10,9 g/dL); anemia moderada (Hb entre 7,0-9,9 g/dL) y anemia severa $(\mathrm{Hb}<7,0 \mathrm{~g} / \mathrm{dL})$. Para la valoración de microcitosis se utilizó el volumen corpuscular medio $<72 \mathrm{fL}$ La ferritina se determinó por el método inmunoenzimático, con una detección final mediante fluorescencia (ELFA), en el equipo miniVidas de BioMérieux. Se consideró valores inferiores a $12 \mu \mathrm{g} / \mathrm{L}$ como indicador de depleción de reservas corporales de hierro para menores de cinco años de edad. Se ajustaron los valores de ferritina según niveles de PCR.

Se evaluó el estado nutricional por indicadores de puntaje z Peso para la Edad, z Talla para la Edad y z Peso para la Talla, según estándares de la Organización Mundial de la Salud.

Se utilizó el programa SPSS 16.0 y el programa Anthro v.3.22 de la Organización Mundial de la Salud.

Fue aprobado por Comité de Ética del Instituto de Medicina Tropical. Se mantuvieron los principios éticos tal como se establece en la Declaración de Helsinki.

\section{RESULTADOS}

En este estudio ingresaron 806 niños. Se determinó estudios laboratoriales y antropométricos en 706 pacientes $(87,6 \%)$.

El promedio de edad de los pacientes fue de $40 \pm 13,1$ meses (12-59 meses). Fueron varones 327 (46.3\%). El $14,9 \%$ correspondió al grupo de lactantes y el $46,5 \%$ fueron de Asunción. (Tabla 1).

Tabla 1. Variables demográficas de los niños de 1 a 4 años que acudieron a Servicios de Salud y Guarderías en Asunción y Central durante el periodo 2017.

\begin{tabular}{lcc}
\hline Edad (meses) & Frecuencia $(\mathbf{N}=\mathbf{7 0 6})$ & \% \\
\hline 12 a 23 & 105 & 14,9 \\
24 a 35 & 161 & 22,8 \\
36 a 47 & 194 & 27,5 \\
48 a 59 & 246 & 34,8 \\
\hline Sexo & & \\
Femenino & 379 & 53,7 \\
Masculino & 327 & 46,3 \\
\hline Procedencia & & \\
Asunción & 328 & 46,5 \\
Central & 378 & 53,5 \\
\hline
\end{tabular}

Se resume en la Tabla 2 los valores promedios y desviación estándar del peso al nacer, de los puntajes z de peso para la edad, peso para la talla, talla para la edad y niveles de albúmina.

Tabla 2. Estado nutricional (antropometría en puntaje z y valores de albúmina plasmática) de niños de 1 a 4 años de edad de Asunción y Central ( $n=706)$.

\begin{tabular}{lcc}
\hline $\begin{array}{l}\text { Indicadores del } \\
\text { estado nutricional }\end{array}$ & Promedio & $\begin{array}{c}\text { 土 Desviación } \\
\text { estándar }\end{array}$ \\
\hline Peso al nacer (gramos)z & 3.290 & 456 \\
Peso para la edadz & 0,29 & 1,33 \\
Peso para la tallaz & $-0,38$ & 1,52 \\
Talla para la edad & 0,92 & 1,64 \\
Albúmina g/dL & 4,46 & 0,20 \\
\hline
\end{tabular}

En la Tabla 3 se resumen el promedio y desviación estándar de los indicadores hematimétricos. La media de Hemoglobina fue de 11, 99 g/dL (rango 6$14,8 \mathrm{~g} / \mathrm{dL}$ ). Del grupo de niños con anemia, 44/83 (53\%) fueron microcíticas (VMC $<72 \mathrm{f}$ ). 
El 11,8 \% del grupo estudiado presentó valores de hemoglobina menores a $11 \mathrm{~g} / \mathrm{dL}$. La prevalencia de anemia grave fue de $0,28 \%(n=2)$, anemia moderada del $3,54 \%(n=25)$ y de anemia leve fue del $8 \%(n=56)$.
El 17,56 \% (n=124) del grupo niños estudiados presentó una ferritina inferior a $12 \mathrm{ug} / \mathrm{L}$. Tuvieron PCR negativa 640/706 (90,75\%) pacientes.

Tabla 3. Valores hematimétricos y de ferritina en niños de 1 a 4 años de edad en Servicios de Salud y guarderías de Asunción y Central, 2017 (n=706).

\begin{tabular}{lcc}
\hline $\begin{array}{l}\text { Indicadores } \\
\text { hematimétricos }\end{array}$ & Promedio & $\begin{array}{c} \pm \text { Desviación } \\
\text { estándar }\end{array}$ \\
\hline Hemoglobina g/dL $(\mathrm{Hb}$ g/dL) & 11,99 & 1,03 \\
Hematocrito \% & 37,02 & 2,51 \\
Volumen Corpuscular Medio VCM & 77,87 & 9,25 \\
Concentración Hb Corpuscular Media CHCM & 32,03 & 3,58 \\
Hemoglobina Corpuscular Media MCH & 25,44 & 5,13 \\
Ferritina $\mu \mathrm{g} / \mathrm{L}$ & 32,87 & 34,76 \\
\hline
\end{tabular}

En la Tabla 4 se resume la prevalencia de anemia por grupos de edad. Se observó una prevalencia de anemia del 11,7\% (83/706) en el grupo de niños de 1 a 4 años de edad.

Del grupo de niños con anemia (n=83), el 68,7 \% fue leve, el $28,9 \%$ fue moderada y un 2,4

$\%$ fue severa.
Al analizar por grupos de edad se observó una mayor prevalencia de anemia en el grupo de lactantes, del $39 \%$ (41/105) versus en el grupo de pre escolares que fue del 6,9\% (42/601). Esta diferencia fue estadísticamente significativa, con OR 5,2-14,1 (Test de $\chi 2 \mathrm{p}<0,05)$. Tabla 4

Tabla 4. Prevalencia de anemia en niños de 1 a 4 años de edad en Servicios de Salud y guarderías de Asunción y Central, $2017(n=706)$.

\begin{tabular}{lccccc}
\hline Edad & Niños sin & \multicolumn{3}{c}{ Niños con anemia } & Total \\
\cline { 3 - 5 } & anemia & Leve & Moderada & Severa & \\
\hline 12 a 23 & 64 & 27 & 13 & 1 & $105^{*}$ \\
24 a 35 & 136 & 19 & 5 & 1 & 161 \\
36 a 47 & 184 & 8 & 2 & 0 & 194 \\
48 a 59 & 239 & 3 & 4 & 0 & 246 \\
Total & $\mathbf{6 2 3}$ & $\mathbf{5 7}$ & $\mathbf{2 4}$ & $\mathbf{2}$ & $\mathbf{7 0 6}$ \\
\hline * $\chi 2$ p $<0,05$ & & & & &
\end{tabular}

\section{DISCUSIÓN}

Un estudio auspiciado por el Fondo de las Naciones Unidas para la Infancia UNICEF en 2003, con 227 niños y niñas de entre 1 y 4 años de edad, procedentes de seis localidades (urbana y rural) de los departamentos de Concepción, Caaguazú, Central y Boquerón, encontró una prevalencia de anemia del $22 \%{ }^{(9)}$. En la presente investigación la prevalencia de anemia en niños de igual grupo etario fue menor y esta diferencia podría atribuirse a que todos los niños eran del área urbana. Los lactantes presentaron un peor resultado. Casi la mitad de los niños entre el año y los dos años de edad presenta anemia. En el trabajo realizado por Echagüe et al, la prevalencia de anemia fue mayor, pero el grupo de estudio estuvo constituido por niños indígenas y niños pertenecientes al área rural ${ }^{(10)}$.

La alta prevalencia de anemia en los lactantes mayores amerita la implementación de políticas y programas. Se podría considerar el ejemplo del caso 
chileno, que lograron disminuir la prevalencia de anemia en lactantes y pre escolares a través de acciones tales como la suplementación con sulfato ferroso al grupo de lactantes, la fortificación de la leche con hierro, zinc y cobre y una amplia cobertura en agua potable y saneamiento básico ${ }^{(11)}$. La Sociedad Brasilera de Pediatría actualmente recomienda la suplementación con hierro hasta los dos años de edad $^{(12)}$ y la Sociedad Argentina de Hematología hasta los 18 meses de edad ${ }^{(13)}$. Esto difiere de las normas actuales de AIEPI que recomiendan la suplementación con hierro hasta el año de edad. Considerando los trabajos de et al. 'y la presente investigación, sería recomendable prolongar la suplementación de hierro en lactantes mayores hasta los dos años de edad, realizar capacitación de los recursos humanos en los servicios de atención primaria de salud y asegurar los insumos de suplementos de hierro en los consultorios de atención primaria.

Las bajas tasas de inflamación, evaluada por los valores de PCR fueron las esperadas y reportados por otros autores para este grupo de edad ${ }^{(14-16)}$. La PCR es un marcador bioquímico de inflamación y un valor negativo indica que no es necesario ajustar los valores de ferritina para casos de inflamación.

Sería importante continuar esta línea de investigación con otros diseños que permitan la determinación de la causalidad de las asociaciones de factores con anemia. La información sobre el ingreso familiar, el estado de la anemia materna prenatal, el tiempo de ligadura del cordón umbilical, el intervalo de nacimiento y el momento de la alimentación complementaria, son algunos de los factores que podrían integrar el análisis de los factores asociados con la anemia en niños de 6 a 59 meses de edad. En un estudio realizado en Ecuador encontraron que la anemia en niños de 1 a 4 años de edad estuvo asociada a déficit de micronutrientes, bajo peso al nacer y prematurez ${ }^{(17)}$. En Brasil analizaron relación de la prevalencia de anemia en niños de 6 a 59 meses con la educación materna, número de hijos menores de 5 años, disponibilidad de agua segura, edad materna, anemia materna y la edad del niño ${ }^{(18)}$.
Las estrategias encaminadas a la prevención de la anemia resultan de máxima importancia en los niños menores de cinco años de edad, sobre todo en el grupo de lactantes. Algunos de los pilares fundamentales para la prevención de la anemia ferropriva constituyen, por ejemplo, conductas perinatales destinadas a mejorar la reserva de hierro materna, ligadura oportuna del cordón umbilical, una adecuada orientación para fomentar la lactancia materna y una alimentación complementaria adecuada, la suplementación con hierro durante los primeros años de vida y fortalecer los programas $\operatorname{alimentarios}^{(19,20)}$.

\section{CONCLUSIONES}

La prevalencia de anemia en lactantes de Asunción y Central constituye un problema de Salud Pública. En cambio, la prevalencia en pre escolares es leve. Un $15,6 \%$ de los niños presentan baja reserva de hierro.

\section{Contribución de autoría}

\section{Fernando Galeano}

Concepción y diseño del estudio, Redacción del manuscrito, Recolección de los datos, Coordinación local en los centros de salud y guarderías, Asumir la corresponsabilidad de todos los aspectos del manuscrito.

\section{Gabriela Sanabria}

Concepción y diseño del estudio, Redacción del manuscrito, Análisis de datos y resultados, Revisión y aprobación de la versión final, Coordinación local en los centros de salud y guarderías.

\section{Marta Sanabria}

Concepción y diseño del estudio, Redacción del manuscrito, Análisis de datos y resultados, Revisión y aprobación de la versión final, Asumir la corresponsabilidad de todos los aspectos del manuscrito

\section{Aníbal Kawabata}

Concepción y diseño del estudio, Análisis de datos y resultados, Revisión y aprobación de la versión final 


\section{Gloria Aguilar}

Concepción y diseño del estudio, Revisión y aprobación de la versión final

\section{Gladys Estigarribia}

Concepción y diseño del estudio, Recolección de los datos, Coordinación local en los centros de salud y guarderías

\section{Iris Vuyk}

Concepción y diseño del estudio, Recolección de los datos, Coordinación local en los centros de salud y guarderías

\section{Sergio Muñoz}

Concepción y diseño del estudio, Revisión y aprobación de la versión final

\section{Fernando Pizarro}

Concepción y diseño del estudio, Revisión y aprobación de la versión final

\section{REFERENCIAS}

1. World Health Organization. Iron deficiency anemia: assessment, prevention, and control: a Guide for Programme Managers. Geneva: WHO; 2001.

2. World Health Organization. The global prevalence of anaemia in 2011.Geneva: WHO; 2015.

3. Sánchez S. Prevalencia de anemia en niños y niñas entre 12 y 60 meses de edad en comunidades seleccionadas. Pediatr. (Asunción). 2004;31:74.

4. Olivares M, Walter T. Consecuencias de la deficiência de hierro. Rev. chil. nutr.. 2003; 30(3):226-233. doi: http://dx.doi.org/10.4067/S0717-75182003000300002

5. Falkingham M, Abdelhamid A, Curtis P, FairweatherTait S, Dye L, Hooper L. The effects of oral iron supplementation on cognition in older children and adults: a systematic review and meta-analysis. Nutr J. 2010;9:4. doi: http://dx.doi.org/10.1186/1475-2891-9-4

6. Pollit E. Effects of a diet deficient in iron on the growth and development of preschool and school - age children. Food and Nutrition Bulletin. 1991; 13(2). doi: https://doi.org/10.1177/156482659101300222

7. Plessow R, Arora NK, Brunner B, Tzogiou C, Eichler K, Brugger U, et al. Social Costs of Iron Deficiency Anaemia in 6-59-Month-Old Children in India. PLoS ONE. 2015;10(8): e0136581.https://doi.org/10.1371/journal.pone.0136581

8. Ministerio de Salud Pública y Bienestar Social. Atención Integrada a las Enfermedades Prevalentes de la Infancia: Manual para profesionales de la salud. Asunción: AIEPI; 2020.
9. Sánchez S, Rojas G, Arellano C, Otero H. Prevalencia de anemia en niños y niñas entre 12 y 60 meses de edad en comunidades seleccionadas. Paraguay: UNICEF; 2004.

10. Echagüe G, Sosa L, Díaz V, Funes P, Ruíz I, Pistilli N. Anemia en niños indígenas y no indígenas menores de 5 años de comunidades rurales del Departamento de Caazapá. Pediatr (Asunción). 2013;40(1):19-28.

11. Hertrampf E, Olivares M, Brito A, Castillo Camiglia A. Evaluación de la prevalencia de anemia ferropriva en una muestra representativa de la Región Metropolitana y Quinta Región de los beneficiarios del Programa Nacional de Alimentación Complementaria PNAC. Santiago; 2009.

12. Fisberg M, Lyra I, Weffort V. Consenso sobre anemia ferropriva: mais que um consenso, uma emergência médica. Rio de Janeiro: Sociedade Brasileira de Pediatria; 2018.

13. Sociedad Argentina de Hematología. Guías de diagnóstico y tratamiento. Buenos Aires: SAH; 2019.

14. Victora CG, Bahl R, Barros AJ, França GV, Horton S, Krasevec J, et al. Breastfeeding in the 21st century: epidemiology, mechanisms, and lifelong effect. The Lancet. 2016;387(10017):475-90. doi: https://doi.org/10.10 16/S0140-6736(15)01024-7

15. Hlaing LM, Fahmida U, Htet MK, Firmansyah A, Utomo B, Gibson RS. Iron Supplementation with or without Optimized Complementary Feeding Recommendations: Effect on Micronutrient Status and Growth of 1-2 Year Old Myanmar Children. European Journal of Nutrition \& Food Safety. 2015;5(5):953-4. doi: https://doi.org/10.9734/EJNFS/2015/21182 
16. Jones AD, Ickes SB, Smith LE, Mbuya MN, Chasekwa B, Heidkamp RA, et al. World Health Organization infant and young child feeding indicators and their associations with child anthropometry: a synthesis of recent findings. Matern Child Nutr. 2014;10(1):1-17. doi: https://doi.org/ 10.1111/mcn.12070

17. Moyano E, Calderon B, Parra C, Ayora E. Factores asociados a la anemia en niños ecuatorianos de 1 a 4 años. Archivos Venezolanos de Farmacología y Terapéutica. 2019; 38(6): 695-699.

18. Leal LP, Batista Filho M, Lira PI, Figueiroa JN, Osório MM. Prevalence of anemia and associated factors in children aged 6-59 months in Pernambuco, Northeastern Brazil. Rev Saude Publica. 2011;45(3):457-66. doi: https://doi.org/10.1590/s0034-89102011000300003
19. Morais Lopez A, Dalmau Serra J. Importancia de la ferropenia en el niño pequeño: repercusiones y prevención. An Pediatr. 2011; 74(6):415.e1-415.e10. doi: https://doi.org/10.1016/j.anpedi.2011.01.036

20. Sociedad Argentina de Pediatría, Comité Nacional de Hematología, Oncología y Medicina Transfusional, Comité Nacional de Nutrición. Deficiencia de hierro y anemia ferropénica. Guía para su prevención, diagnóstico y tratamiento. Arch Argent Pediatr .2017; 115(Supl 4):s68-s82. 